\title{
Use The Positive Selection Algorithm and The Negative Selection Algorithm in The English Character Recognition
}

\author{
Maha A.M. Hassan \\ College of Computer \\ Science and \\ Mathematics/ \\ Department of Software \\ Engineering \\ mahahassan@gmail.com \\ DOI: 10.33899/edusj.1970.163331

\section{Received} \\ 23/ $10 / 2013$
}

\author{
Maha H. Najam \\ College of Computer \\ Science and \\ Mathematics/ \\ Department of Software \\ Engineering \\ mahanajam@gmail.com \\ Accepted \\ 05 / 03 / 2014
}

\author{
Nour S. Shiban \\ College of Computer \\ Science and \\ Mathematics/ \\ Department of Software \\ Engineering \\ nourshiban@gmail.com
}




\section{استخدام خوارزمية الانتقاء الايجابي وخوارزمية الانتقاء السلبي في \\ تمييز الحروف الانكليزية}

$$
\begin{aligned}
& \text { مها حمادي نجم } \\
& \text { كلية علوم الحاسوب } \\
& \text { والرياضيات/ } \\
& \text { قسم هندسة البرمجيات }
\end{aligned}
$$

nourshiban@gmail.com mahanajam@gmail.com DOI: $\underline{10.33899 / \text { edusj.1970.163331 }}$

$$
\text { كلية علوم الحاسوب علفتاح }
$$$$
\text { قسم هندسة البرمجيات }
$$

nourafattah@gmail.com

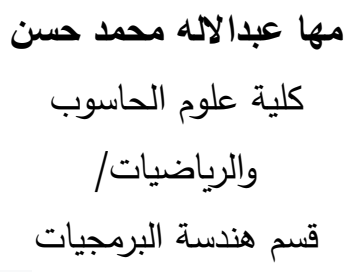

mahahassan@gmail.com

\section{الاستلام}

$2014 / 03 / 05$

$2013 / 10 / 23$

الملخص

تم في هذا البحث استخدام بعض من خوارزميات التقنيات الذكائية الاصطناعية وهي خوارزميات النظام المناعي الاصطناعي (خوارزمية الانتقاء الايجابي وخوارزمية الانتقاء السلبي) حيث تم اجراء عملية تمييز بين الحروف الانكليزية الاصلية وبين الحروف

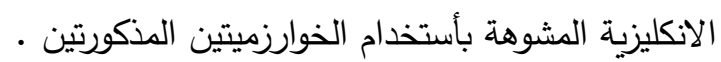

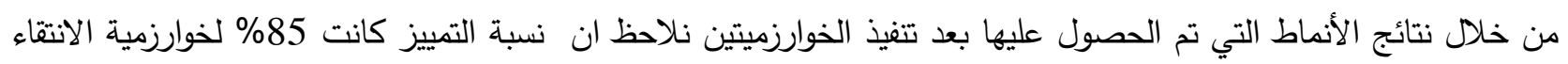

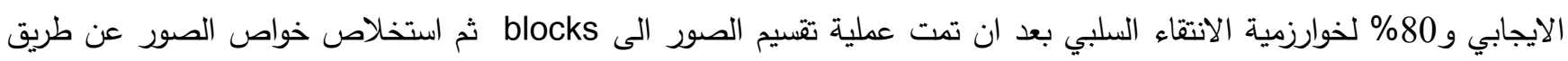

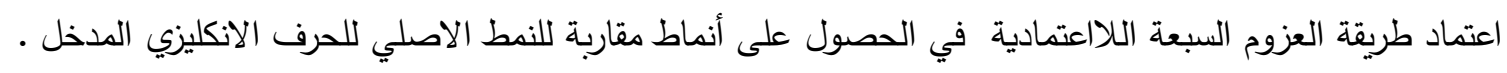

الكلمات المفتاحية: نظام المناعة الأصطناعي , استخلاص الخواص ,خوارزمية الانتقاء الايجابي وخوارزمية الانتقاء السلبي.

1

إن أتمتة الخط كانت وما زالت مجالا مهما منذ بدايات الحاسبات. ففي العام 1972 أظهر مسحاً اجري ما يقارب 130 عملا في هذا المجال[1] , وبالرغم من قدم الموضوع إلا أن تمييز الحروف ما زال واحداً من أكثر المجالات تحديا وإثارة من مجالات البحث في علوم الحاسبات, إذ تطور هذا المجال في السنوات الأخيرة إلى هيكل ناضج ومنتج لأكثر الأعمال وأفضلها. فعلى الرغم من التتبؤات

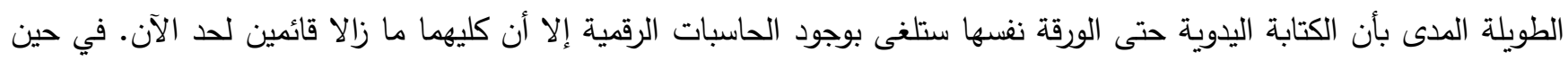
سهّل الحاسوب عملية إنتاج الوثائق المطبوعة بشكل كبير , إلا أن الراحة والسهولة التي تمنحهما الورقة والقلم تجعلهما دائما الوسيلة الطبيعية لإنجاز الكثير من المهام[2] ـ 
بسبب تطبيقات النظام المناعي AIS الواسعة والفعالة وخصوصا في مجال تمييز الأنماط pattern recognition, فقد تم تنفيذ مرحلة

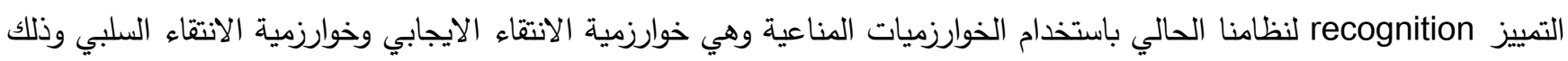

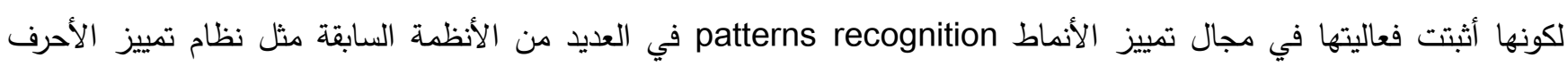
الهندية [3].

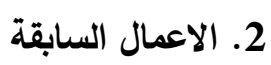

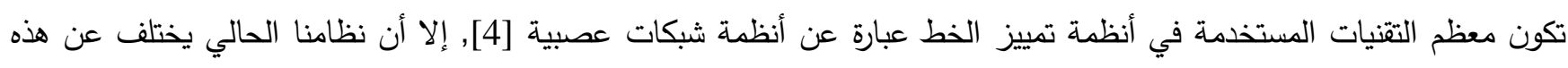
الأنظمة, وذلك لكون الأنظمة المعتدة على الثبكات العصبية في التمييز عادة ما تحتاج إلى عدد كبير من النماذج التي تحتئ تحتاج إليها للتدريب training فضلاً عن الوقت الكبير الذي تحتاجه هذه الأنظمة في التدريب من أجل تحقيق نسبة تمييز جيدة, أما بالنسبة لبحثنا

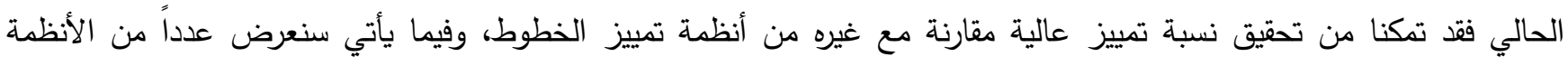

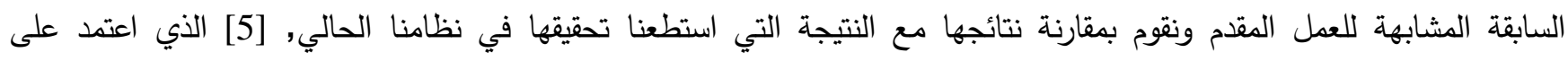

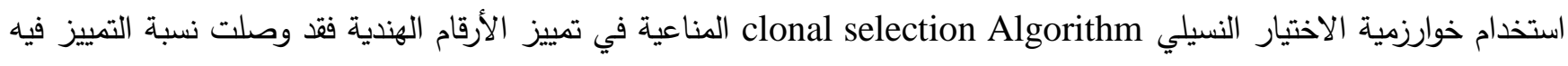

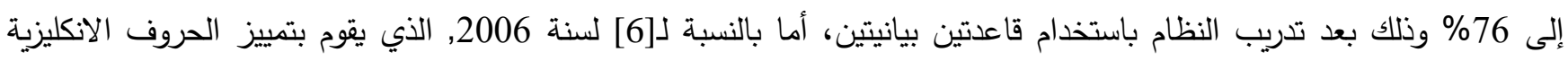

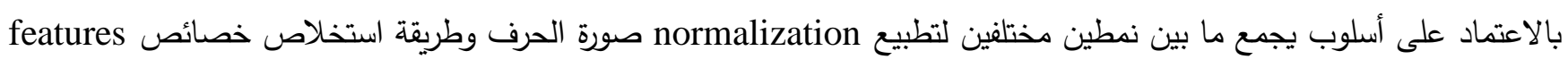

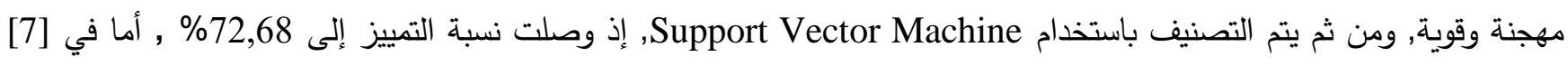
لسنة 2009, تم تنفيذ نظام لتمييز الحروف الانكليزية باستخدام الشبكات العصبية وقد تم تحقيق معدل نسبة تمييز وصل إلى باستخدام نماذج تدريب وصلت إلى 592 ونماذج اختبار وصلت إلى 825 نموذجاً.

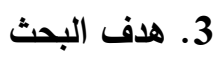

يهدف البحث الى اجراء عملية تمييز صورة الحرف الانكليزي المشوه حاسوبياً (فقدان جزء من الحرف )على سبيل المثال الحرف الحف

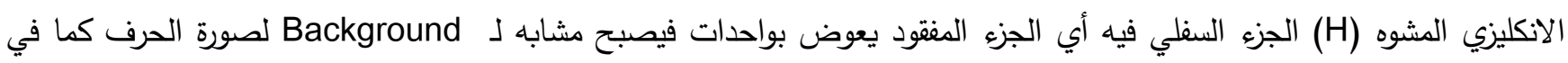

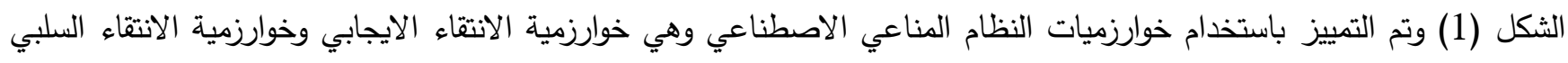

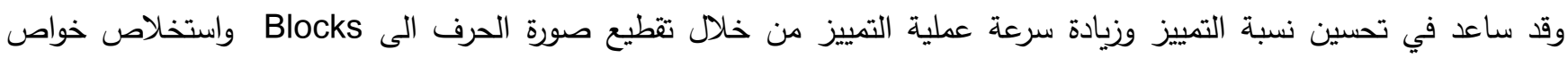
الصورة عن طريق حساب العزوم السبعة لقطع الحرف الانكليزي الاصلي والمشوه.

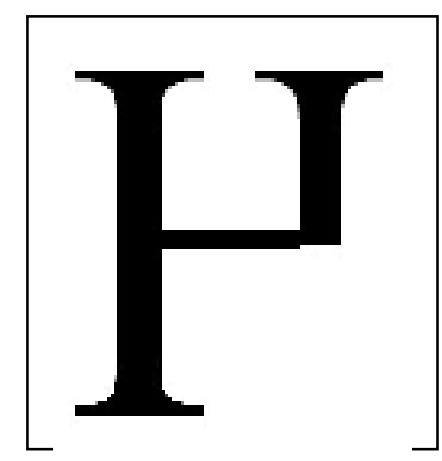

الشكل (1) يمثل صورة الحرف الانكليزي (H) المشوه حاسوبياً 
هو منهج متطور حديث مستوحى من جهاز المناعة الطبيعي في الإنسان, ظهر في التسعينات على أنه فرع جديد من الحساب التطوري Evolutionary computation [8] ان جهاز المناعة الطبيعي معقد جدآ ليتم محاكاته صناعيا, ولكن نجح A.B.Watkins بمحاكاة أهم وظائف جهاز المناعة الطبيعي فيما يخص تمييز الأنماط، إن نظام المناعة الاصطناعي هو مجموعة

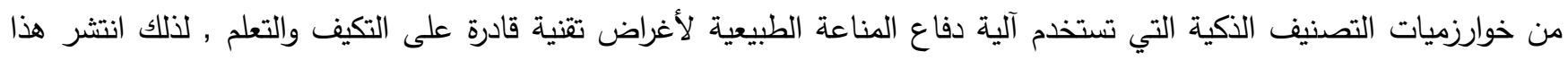
المفهوم في تطبيقات تقنية عديدة خلال العقود الأخيرة [9] ، إن الحوسبة المستخدمة أحيائياً(Biologically Inspired Computing) وخصوصا أنظمة المناعة الاصطناعية (AIS) هي حل واعد لتطوير أدوات دفاعية مكيفة ومؤتمتة للتهديدات الحالية والمستقبلية في

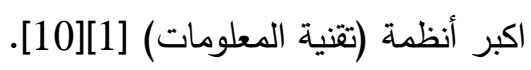
قدم De.Castro Timmis و إطار عمل مستخدم بصورة أكثر شيوعا في هندسة AIS يتضمن أطار العمل ثلاث خطوات مستقلة بشكل نسبي في بناء) (AIS) أن أساس كل نظام هو مجال التطبيق [11], ويعمل تمثيل البيانات على التعريف بكيفية تحويل بيانات التطبيق الملاحظة إلى بيانات متلائمة النسق وذلك ليتم معالجتها بواسطة خوارزميات( AIS) تحتاج البيانات المستخدمة أن تكون

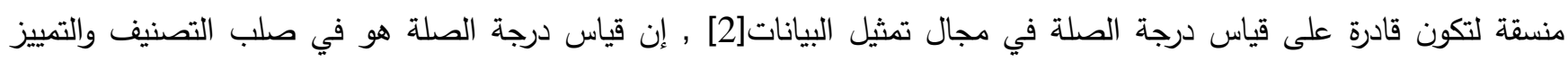

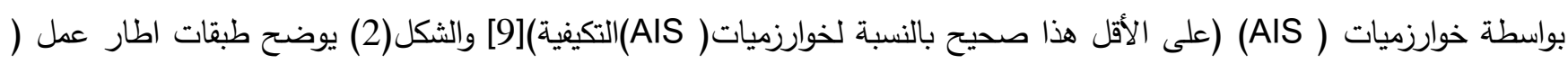

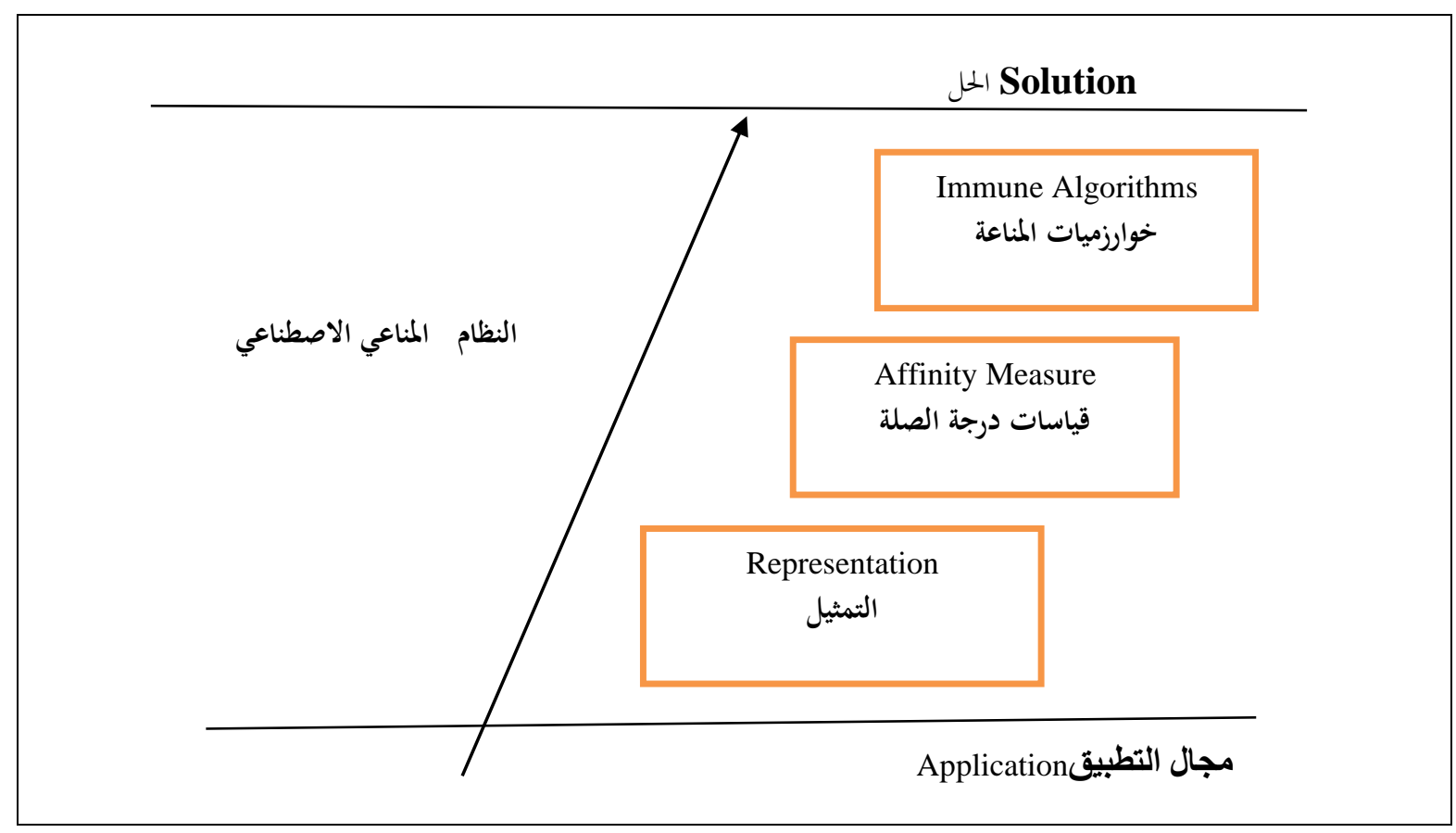

[8](AIS ) الشكل (2) يوضح طبقات اطار عمل 
يمتلك جهاز المناعة الاصطناعي ( AIS) عدة صفات حسابية مميزة، وفيما يلي بعض منها [12] [13]: 1. تمييز الأنماط (Pattern Recognition) ): يستطيع جهاز المناعة أن يميز مستضدات معينة ويولد استجابات مناسبة حيث يتم انجاز ذلك بواسطة آلية التمييز المعتمدة على الارتباط للمستقبلات و المستضدات. يعتمد هذا الارتباط على أشكالها الجزيئية و الإثارات[13].

2. استخلاص الخواص (Feature Extraction) : بصورة عامة لا ترتبط مستقبلات المناعة بمستضد كامل بل أجزاء منه ووفقا إلى ذلك يمكن لجهاز المناعة أن يميز مستضدآ فقط عن طريق التطابق مع أجزاء منه[7].

\section{5eature Extraction 5. استخلاص الخواص}

إنّ عملية استخلاص الخواص واحدة من أهم عناصر نظام تمييز الانماط ، إذ يعتد أداء نظام التصنيف بشكل مباشر على عملية اختيار الخواص و استخلاصها][6]، الفائدة الرئيسة من استخلاص الخواص هي تقليص حجم البيانات المعروضـة بالصورة وتمثل الصورة بجموعة من الخواص بصيغة رقمية وهذه الخواص تستخدم في نظام التمييز لتصنيف البيانات وتجعل خوارزمية استخلاص الخواص في عملية التمييز أكثر فاعلية و أعلى كفاءة ، إذ أنّ نظام التمييز يتعامل مع مفاهيم رياضية و تقنية من أجل تصنيف

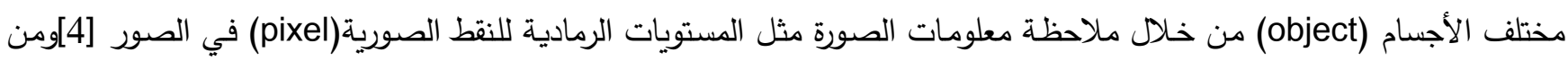
الحسابات الاحصائية المستخدمة في استخلاص الخواص هي العزوم اللاعتمادية (Moment invariants) تحسب هذه العزوم باستعمال مجموعة لا خطية من اللحظات الهنسية (Moment geometric) والتي لها الإمكانية المطلوبة بأنها ثابتة (invariant) بعد ازاحة الصورة او تكبيرها اوتصغيرها او تدويرها وهناك مجموعة عزوم مركزية (Central Moments) لصورة ثنائية البعد ممكن إيجادها بخطوات عدة:

$m_{p q}=\sum \sum x^{p} y^{q} f(x, y)$

الخطوة الأولى : حساب العزوم الثلاثة الأولى من المعادلة الآتية:[11

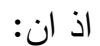
Mpq $1,0: p, q$ $\bar{x}=\frac{m_{10}}{m_{00}}$ And $\bar{y}=\frac{m_{01}}{m_{00}}$

$\mu_{p q}=\sum_{x} \sum_{y}(x-\bar{x})^{p}(y-\bar{y})^{q} f(x, y)$ 
Mpq

$$
\begin{aligned}
& \mu_{00}=\sum_{x} \sum_{y}(x-\bar{x})^{0}(y-\bar{y})^{0} f(x, y)=\sum_{x} \sum_{y} f(x, y)=m_{00} \\
& \mu_{10}=\sum_{x} \sum_{y}(x-\bar{x})^{1}(y-\bar{y})^{0} f(x, y)=m_{10}-\frac{m_{10}}{m_{00}}\left(m_{00}\right)=0 \\
& \mu_{01}=\sum_{x} \sum_{y}(x-\bar{x})^{0}(y-\bar{y})^{1} f(x, y)=m_{01}-\frac{m_{01}}{m_{00}}\left(m_{00}\right)=0 \\
& \mu_{11}=\sum_{x} \sum_{y}(x-\bar{x})^{1}(y-\bar{y})^{1} f(x, y)=m_{11}-\frac{m_{10} m_{01}}{m_{00}}=m_{11}-\bar{y} m_{10} \\
& \mu_{20}=\sum_{x} \sum_{y}(x-\bar{x})^{2}(y-\bar{y})^{0} f(x, y)=m_{20}-\frac{2 m_{10}^{2}}{m_{00}}+\frac{m_{10}^{2}}{m_{00}}=m_{20}-\frac{m_{10}^{2}}{m_{00}}=m_{20}-\bar{x} m_{10} \\
& \mu_{02}=\sum_{x} \sum_{y}(x-\bar{x})^{0}(y-\bar{y})^{2} f(x, y)=m_{02}-\frac{m_{01}^{2}}{m_{00}}=m_{02}-\bar{y} m_{01} \\
& \mu_{21}=\sum_{x} \sum_{y}(x-\bar{x})^{2}(y-\bar{y})^{1} f(x, y)=m_{21}-2 \bar{x} m_{11}-\bar{y} m_{20}+2 \bar{x}^{2} m_{01} \\
& \mu_{12}=\sum_{x} \sum_{y}(x-\bar{x})^{1}(y-\bar{y})^{2} f(x, y)=m_{12}-2 \bar{y} m_{11}-\bar{x} m_{02}+2 \bar{y}^{2} m_{10} \\
& \mu_{30}=\sum_{x} \sum_{y}(x-\bar{x})^{3}(y-\bar{y})^{0} f(x, y)=m_{30}-3 \bar{x} m_{20}+2 \bar{x}^{2} m_{10} \\
& \mu_{03}=\sum_{x} \sum_{y}(x-\bar{x})^{0}(y-\bar{y})^{3} f(x, y)=m_{03}-3 \bar{y} m_{02}+2 \bar{y}^{2} m_{01}
\end{aligned}
$$

والعزوم المركزية التطبيعية (Normalized Central Moments) تحسب كما في المعادلة الآتية:

$$
\begin{gathered}
\eta_{p q}=\frac{\mu_{p q}}{\mu_{\mathrm{oO}}^{r}} \\
r=\frac{p+q}{2}+1
\end{gathered}
$$

من المعادلات الآتية تحسب العزوم السبعة بالاعتماد على قيم العزوم المركزية التطبيعية : 


$$
\begin{aligned}
\phi_{1}= & \eta_{20}+\eta_{02} \\
\phi_{2}= & \left(\eta_{20}-\eta_{02}\right)^{2}+4 \eta_{11}^{2} \\
\phi_{3}= & \left(\eta_{30}-3 \eta_{12}\right)^{2}+\left(3 \eta_{21}-\eta_{03}\right)^{2} \\
\phi_{4}= & \left(\eta_{30}+\eta_{12}\right)^{2}+\left(\eta_{21}+\eta_{03}\right)^{2} \\
\phi_{5}= & \left(\eta_{30}-3 \eta_{12}\right)\left(\eta_{30}+\eta_{12}\right)\left[\left(\eta_{30}+\eta_{12}\right)^{2}-3\left(\eta_{21}+\eta_{03}\right)^{2}\right] \\
& +\left(3 \eta_{21}-\eta_{03}\right)\left(\eta_{21}+\eta_{03}\right)\left[3\left(\eta_{30}+\eta_{12}\right)^{2}-\left(\eta_{21}+\eta_{03}\right)^{2}\right] \\
\phi_{6}= & \left(\eta_{20}-\eta_{02}\right)\left[\left(\eta_{30}+\eta_{12}\right)^{2}-\left(\eta_{21}+\eta_{03}\right)^{2}\right]+4 \eta_{11}\left(\eta_{30}+\eta_{12}\right)\left(\eta_{21}+\eta_{03}\right) \\
\phi_{7}= & \left(3 \eta_{21}-\eta_{03}\right)\left(\eta_{30}+\eta_{12}\right)\left[\left(\eta_{30}+\eta_{12}\right)^{2}-3\left(\eta_{21}+\eta_{03}\right)^{2}\right] \\
& +\left(3 \eta_{12}-\eta_{30}\right)\left(\eta_{21}+\eta_{03}\right)\left[3\left(\eta_{30}+\eta_{12}\right)^{2}-\left(\eta_{21}+\eta_{03}\right)^{2}\right]
\end{aligned}
$$

بعد ان يتم تحليل صورة الحرف الانكليزي الاصلي والمشوه من خلال استخلاص خواص صورة الحرف عن طريق حساب العزوم السبعة

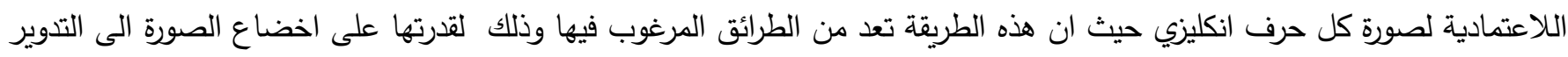

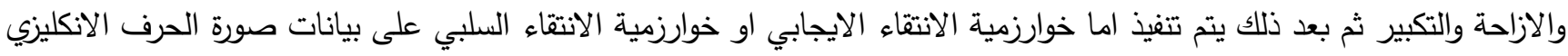
لغرض تمييز هذه الحروف .

\section{positive selection algorithm and negative selection algorithm}

في عام 1992[ 14] اقتُرح نموذج اتمته خلوية لإنجاز محاكاة حاسوبية للجهاز المناعي الاصطناعي سميت بخوارزمية الانتقاء الايجابي حيث تمتلك الخوارزمية خصائص مفيدة من منظور حسابي، الخطوات الاساسية للخوارزمية هي: المُدخل :T Tseen = مجموعة من العناصر الذاتية المعلومة. المُخرج : G = مجموعة المستكثفات المتولدة. أبدأ(begin) كرر (repeat)

$$
\text { - ولَّد مستكشفات بشكل عشوائي وضعها في مجموعة }
$$

- حدد دالة اللياقة لكل عنصر (عضو) في P مع كل عنصر في الدجموعة الذاتية

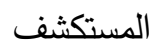

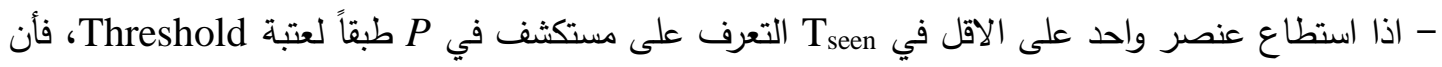

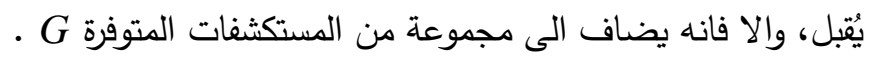
(until) انتهى(end 


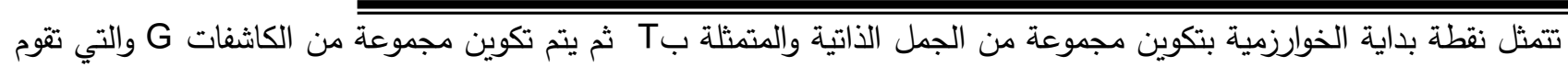
بتمييز متمم T فقط ثم يتم تطبيق هذه الكاشفات على بيانات جديدة من اجل تمييزها، في عملنا المقدم فان الرمز Tseen في الخوارزمية يقابل

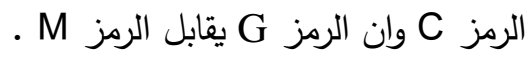

تعد نظرية الانتقاء السلبي فكرة مفيدة جداً لتطوير الخوارزميات التي تراقب الانظمة ضد وقوع حالة الثذوذ او السلوك الغير مألوف [8], ان السمة المفيدة لهذه الخوارزمية هي امكانية استعمالها في اداء المهمات مثل تمييز الانماط وتمييز الصور والامثلية، الخطوات الاساسية للخوارزمية هي: المُدخل : S S S S S المُخرج : D = مجموعة المستكشفات المتولدة. أبدأ(begin)

كرر (repeat)

- ولّد مستكثفات بشكل عشوائي وضعها في مجموعة

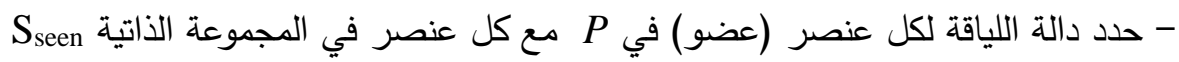

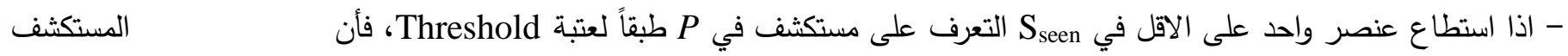

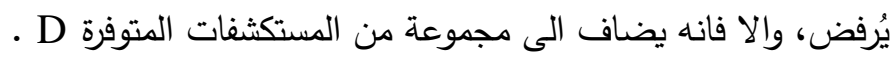
(until) (end) انتهى

تتمثل نقطة بداية الخوارزمية بتكوين مجموعة من الجمل الذاتية والمتمثلة بS ثم يتم تكوين مجموعة من الكاشفات D والتي تقوم بتمييز متمم S فقط ثم يتم تطبيق هذه الكاشفات على بيانات جديدة من اجل تمييزها، في عملنا المقدم فان الرمز S S في الخوارزمية يقابل

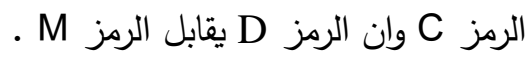
لحساب قيمة الالت التي تمثل درجة الصلة بين صورة الحرف الانكليزي الاصلي والحرف الانكليزي المشوه لكلا Affinity $=\frac{X \$ 100}{Y}$ الخوارزميتين تحسب بالشكل التالي: حيث ان:

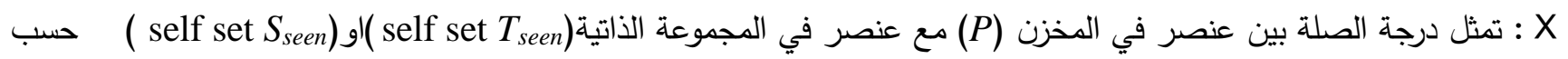
نوع الخوارزمية المستخدمة. ت : تمثل عدد صور الحروف الانكليزية المشوهة المدخلة في النظام حيث ان هذه القيمة قابلة للتغيير حسب حاجة المستخدم للنظام • 7. الجانب العملي للبحث يعتبر النظام المناعي الاصطناعي احدى اهم التقنيات الذكائية الاصطناعية المستخدمة في مجال تمييز الانماط واستخلاص الخواص حيث تم في البحث استخدام خوارزمية الانتقاء السلبي وخوارزمية الانتقاء الايجابي في عملية تمييز انماط الحروف الانكليزية المشوهه علماً ان الحروف الانكليزية الاصلية والمشوهة مكتوبة بخط نوعه(Times New Roman) وحجم الخط (18) حيث تم أدخال الصورة 
عن طريق الماسح الضوئي وحفظت الصورة بصيغة(bmp) وبعدها تم تقطيع الصورة وحساب العزوم السبعة ثم اجراء عملية المقارنة

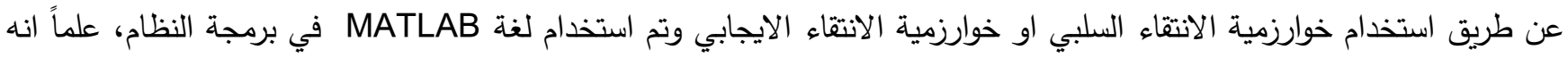

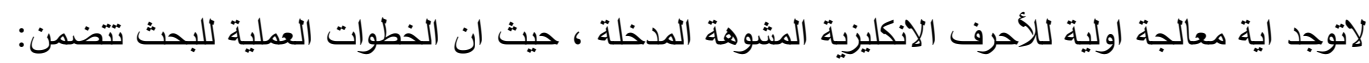

الخطوة الاولى:ادخال مجموعة من الاحرف الانكليزية الصحيحة والمشوهة عن طريق الماسح الضوئي(إ(Scanner).

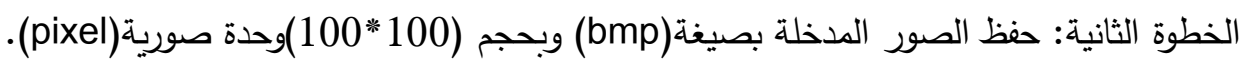
الخطوة الثالثة: يتم تقطيع الصور الدذخلة الى عدة(Blocks). الخطوة الرابعة: يتم حساب العزوم السبعة لكل (Block) عن طريق استخلاص خواص الصناص الصور .

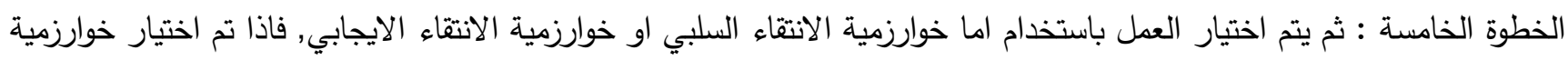
الانتقاء الايجابي فيتم اتباع الخطوات التالية في البرنامج:

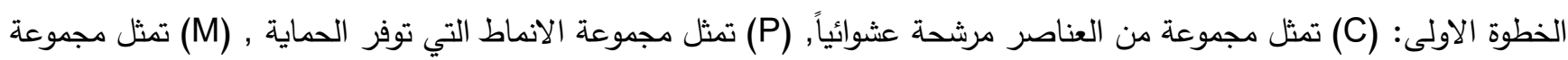

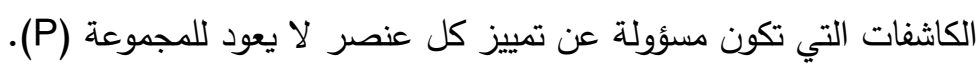
الخطوة الثانية: المقارنة بين العناصر في (C) والعناصر في (P) من ناحية مقدار الصلة فيما بينهم:

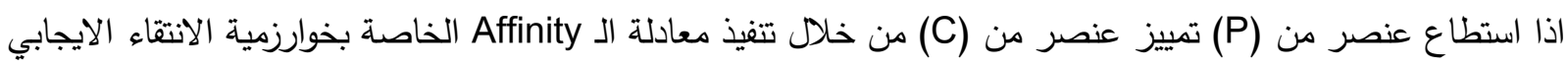
وهي كالتالي:

Affinity $=\frac{X * 100}{64}$

حيث ان :

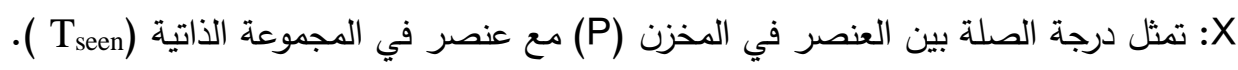

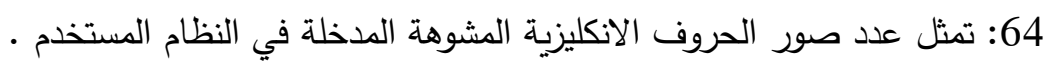
اذا كانت قيمة ال Affinity الناتجة اكبر او مساوٍ لحد العتبة Threshold (قيمة حد العتبة تحدد حسب صورة كل حرف فئن

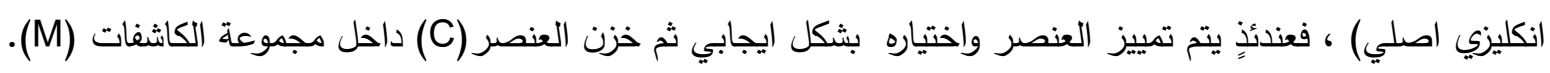

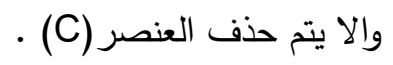

وهكذا تتكرر الخطوات حتى يتم التمييز بين العناصر.

اما اذا تم اختيار خوارزمية الانتقاء السلبي فيتم اتباع الخطوات التالية:

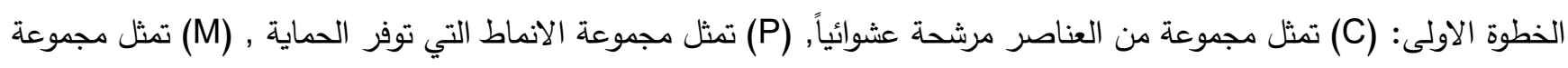

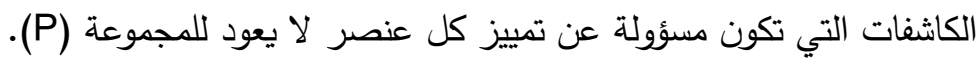

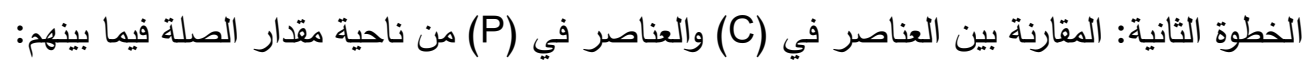
اذا استطاع عنصر من (P) تمييز عنصر من (C) من خلال تنفيذ معادلة الـ Affinity الخاصة بخوارزمية الانتقاء السلبي وهي كالتالي: 
Affinity $=\frac{X * 100}{64}$

64: تمثل درجة الصلة بين العنصر في المخزن (P) مع عنصر في المجموعة الذاتية (S S ( ).

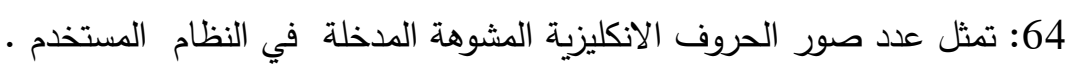

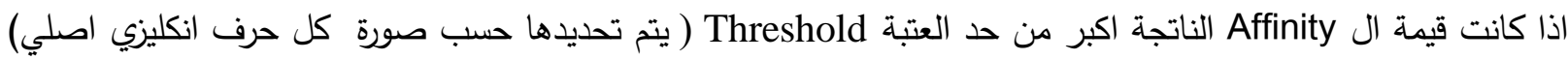

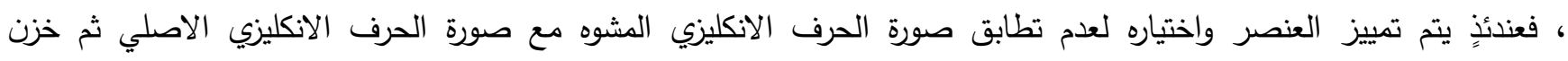

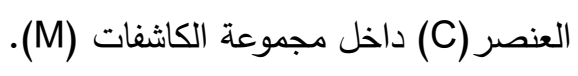

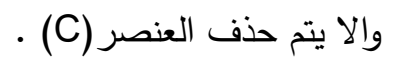
وهكذا تتكرر الخطوات حتى يتم التمييز بين العناصر .

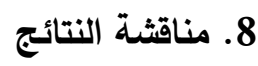
من خلال تطبيق النظام لغرض تمييز الحروف الانكليزية المشوه وعلى وفق النتائج التي تم الحصول عليها، تم التوصل إلى

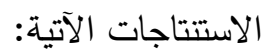
1. بعد دراسة الخوارزميات الموجودة في حقل أنظمة AIS تم اختيار خوارزمية الاختيار الانتقاء الايجابي وخوارزمية الانتقاء السلبي لبناء الحل للمشكلة المدروسة لما فيها من قابليات مناسبة لاداء بعض المهام مثل التعلم باستخدام الحاسبة وتمييز الانماط وتمييز

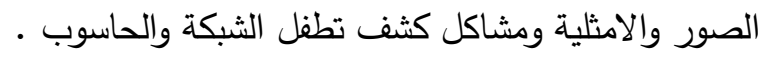

$$
\text { تم تحقيق نسب التمييز الآتية: }
$$

\begin{tabular}{|c|c|}
\hline نسبة التمييز & نوع الإدخال \\
\hline بلغت النسبة & فوارزمية الانتقاء الايجابي تور الحروف الإنكليزية المشوهة باستخدام \\
\hline بلغت النسبة & فوارزمية الانتقاء السلبي صور الحروف الإنكليزية المشوهة باستخدام \\
\hline
\end{tabular}

3. تم تكوين قاعدة بيانات تضم صور للحروف الانكليزية المشوهة تحتوي على 64 نموذج بالإضافة الى 26 نموذج من صور

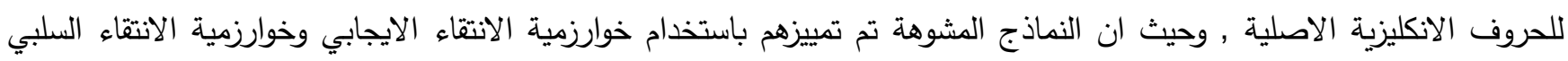

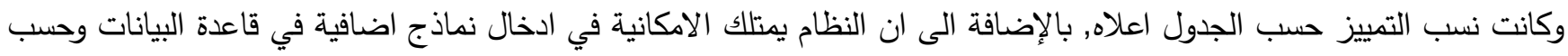
حاجة المستخدم للنظام. 


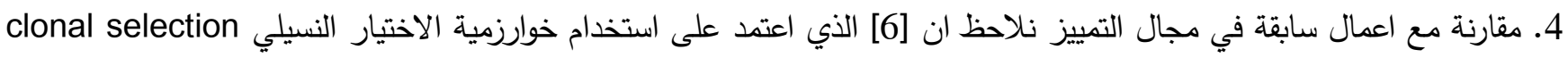
المناعية في تمييز الأرقام الهندية فقد وصلت نسبة التمييز فيه إلى 76\% وذلك بعد تدريب النظام باستخدام قاعدتين بيانيتين أما في

[3] لسنة 2009 تم تتفيذ نظام لتمييز الحروف الانكليزية باستخدام الثبكات العصبية وقد تم تحقيق معدل نسبة تمييز وصلت إلى إلى

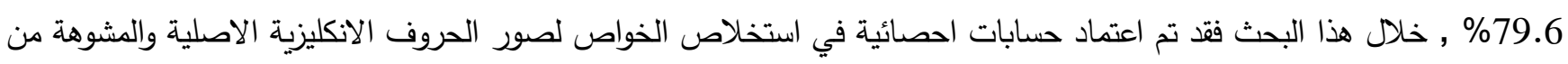
خلال اعتماد طريقة العزوم السبعة اللاعتمادية لقدرتها على تعديل الصور من خلال التكبير والازاحة والتدوير , وإن هذه الطريقة مكنة

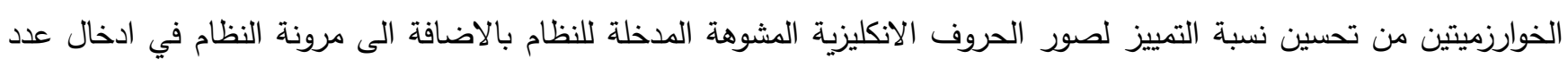
صور الحروف الانكليزية المشوهة وحسب حاجة المستخدم للنظام.

9

من خلال تطبيق النظام المناعي الاصطناعي لغرض تمييز الحروف الانكليزية وعلى وفق النتائج التي تم الحصول عليها، تم التوصل

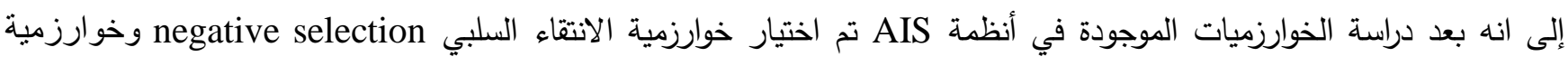
الانتقاء الايجابي positive selection لما لهما من قابليات عالية في معالجة المعلومات وبنمط عالٍ من التوازي والتوزيع في مجال التصنيف وتمييز الانماط وتمييز الصور .

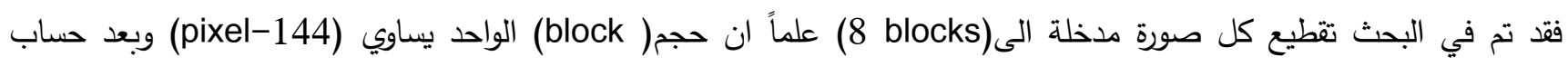
العزوم لكل (blocks) تم الحصول على قيم عزوم كفوية ومناسبة للتمييز , من خلال النتائج التي تم التوصل اليها نلاحظ انه: في خوارزمية الانتقاء الايجابي كلما كانت قيمة الـ Affinity كبيرة لصورة الحرف الانكليزي المشوه مقارنة بقيمة العتبة التابعة لصورة الحرف الانكليزي الاصلي بمعنى ان صورة هذا الحرف الانكليزي المشوه مقاربة اكثر لصورة الحرف الانكليزي الاصلي بالنسبة لبقية صور الحروف الانكليزية المشوهة عندئذٍ يتم اختيار هذه الصورة كأفضل صورة ناتجة, كما في الشكل(3).

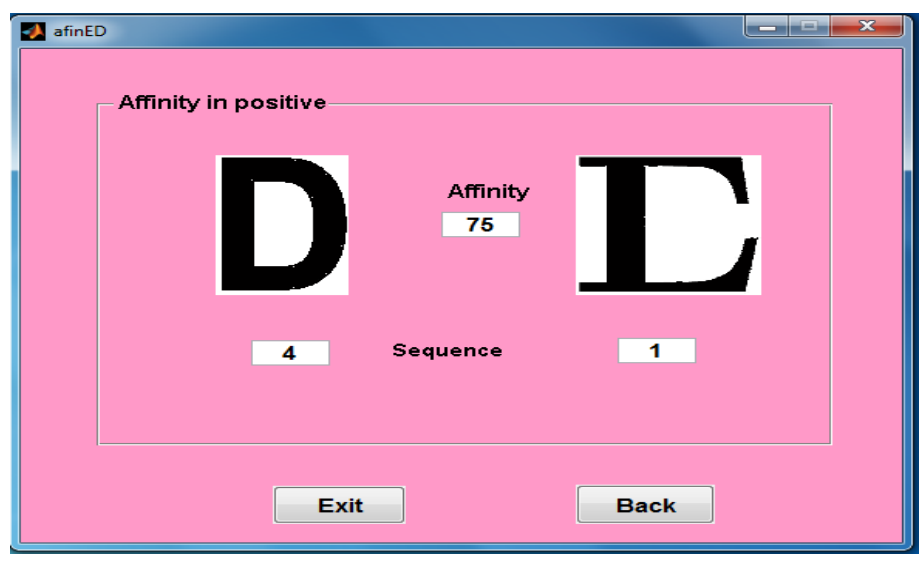

الثكل(3) يوضح اعلى نسبة تطابق لصورة الحرف الانكليزي المشوه (D)

$$
\text { مع صورة الحرف الانكليزي الاصلي(D) في خوارزمية الانتقاء الايجابي تلطياي }
$$




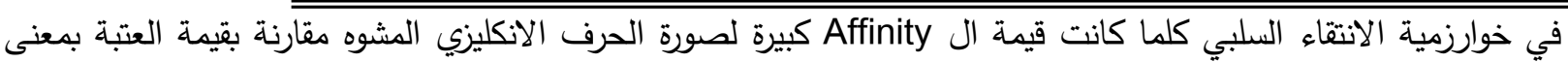

ان صورة هذا الحرف الانكليزي المشوه مختلفة (ليست مقاربة) تماماً لصورة الحرف الانكليزي الاصلي بالنسبة لبقية صور الحروف الانكليزية المشوهة عندئذٍ يتم اختيار هذه الصورة كأفضل صورة ناتجة من الخوارزمية كما في الشكل(4).

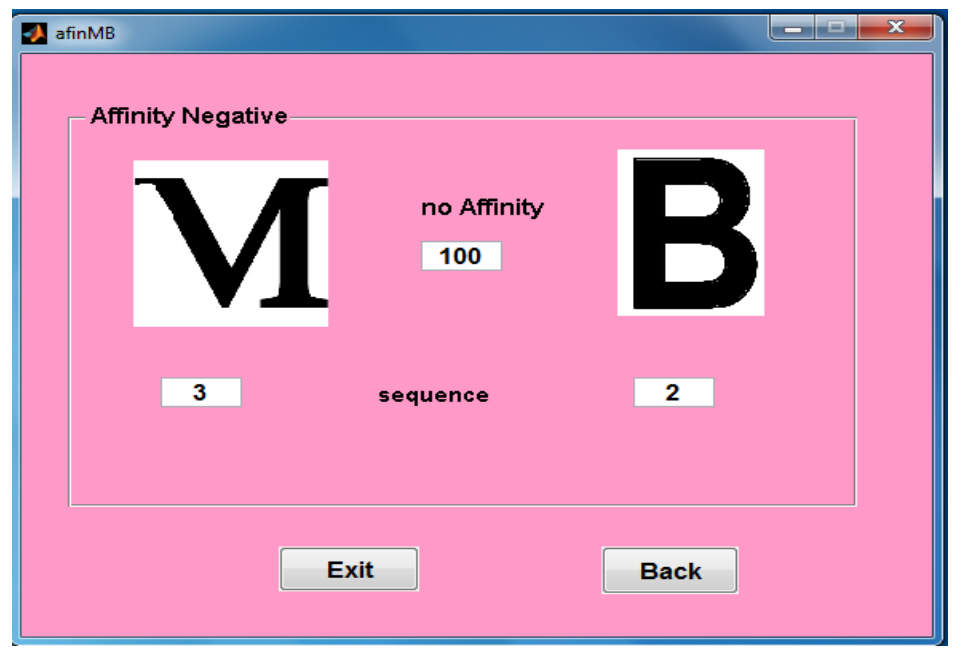

الثكل(4) يوضح اعلى نسبة اختلاف (عدم تشابه) لصورة الحرف الانكليزي الششوه (M)

مع صورة الحرف الانكليزي الاصلي(B) في خوارزمية الانتقاء السلبي

إن استخدام خوارزمية الاختيار السلبي والايجابي ساعد كثيرا في تحسين نسبة التمييز حيث ان نسبة التمييز 85\%

لخوارزمية الانتقاء الايجابي و80\% لخوارزمية الانتقاء السلبي بالاضافة الى زيادة سرعة عملية التمييز عن طريق تقطيع صور

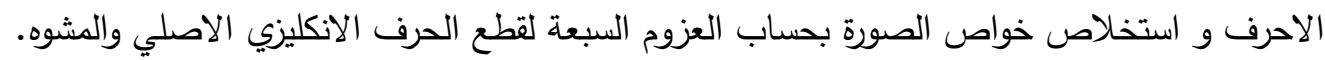

\section{المصادر}

1. Coello, C., Cortez-Rivera, D., Cruz-Cortez, N., (2003), Proc. ICARIS, Springer, pp.1—10.

2. Dasgupta, D., Niño, Luis F., (2009), CRC, ISBN 978-1-4200-6545-9.

3. Goldsby, Richard, A., Kindt, Thomas, J., Osborne, Barbara, A, Kuby, J., (2003), " Immunology ", $5^{\text {th }}$ Edition, W. H. Freeman and Company.

4. Andrew Watkins, Xintong BI and Amit Phadke, 2003, Department of Computer Science and Engineering, Mississippi State University, USA, Also: Computing Laboratory, University of Kent, Canterbury, UK. 
5. De Castro L.N., Von Zuben, F.J., (2002), IEEE Trans. Evol. Comput., vol. 6, no. 3, pp 239251.

6. B. Gatos, I. Pratikakis, A.L. Kesidis and S.J. Perantonis,2006, Computational Intelligence Laboratory, Institute of Informatics and Telecommunications, National Center for Scientific Research "Demokritos", GR-153 10 Agia Paraskevi, Athens, Greece.

7. Divya Sharma, June 2009, Master of Engineering in Software Engineering, Thapar University.

8. Forrest, S., Perelson, A., Allen, L., Cherukuri, R., (1994), Proc. of the IEEE Symposium.

9. De Castro, L. N., Timmis, J., (2002), ISBN 1-85233-594-7, Springer, England.

10. Foster, R. , (2010), PhD thesis, University of Nottingham, England.

11. Haidar Almohri, John S.Gray and Hisham Alnajjar, PhD, 2008.

12. Iqbal, A., (2006), $\mathrm{PhD}$ theises, Universiti Teknologi Malaysia.

13. Greensmith, J., Aickelin, U., Cayzer, S., 2005, In ICARIS-05, LNCS 3627, pp. 153-167.

14. Seiden, P.E. and Celada , F.,(1992), J. theor. Bio., 158,pp.329-357. 